\title{
Partitioning of bronchopulmonary carcinoids in two different prognostic categories by Ki-67 score
}

\author{
Franco Grimaldi ${ }^{1}$, Daniele Muser ${ }^{2}$, Carlo Alberto Beltrami ${ }^{3}$, Piernicola Machin ${ }^{3}$, Angelo Morelli ${ }^{2}$, Stefano \\ Pizzolitto $^{4}$, Giovanni Talmassons ${ }^{2}$, Francesca Marciello ${ }^{5}$, Annamaria Anita Livia Colao ${ }^{5}$, Roberto Monaco ${ }^{6}$, \\ Guglielmo Monaco ${ }^{7}$ and Antongiulio Faggiano ${ }^{8 *}$
}

\author{
Endocrinology and Metabolism Unit, Azienda Ospedaliero-Universitaria Santa Maria della Misericordia di Udine, Udine, Italy \\ ${ }^{2}$ Cardiothoracic Surgery, Azienda Ospedaliero-Universitaria Santa Maria della Misericordia di Udine, Udine, Italy \\ ${ }_{3}$ Pathology Department, Azienda Ospedaliero-Universitaria Santa Maria della Misericordia di Udine, Udine, Italy \\ ${ }^{4}$ Institute of Pathological Anatomy, Azienda Ospedaliero-Universitaria Santa Maria della Misericordia di Udine, Udine, Italy \\ ${ }^{5}$ Department of Molecular and Clinical Endocrinology and Oncology, Federico II University, Napoli, Italy \\ 6 Pathology Unit, Azienda Ospedaliera di Rilievo Nazionale Cardarelli, Napoli, Italy \\ 7 Thoracic Surgery Unit, Azienda Ospedaliera di Rilievo Nazionale Cardarelli, Napoli, Italy \\ ${ }^{8}$ Istituto di Ricovero e Cura a Carattere Scientifico Fondazione SDN, Napoli, Italy
}

\section{Edited by:}

Philippe Chanson, Université

Paris-Sud, France

Reviewed by:

Giovanni Vitale, Universitá Degli Studi di Milano, Italy

Arantzazu Sebastian-Ochoa, Virgen de la Victoria Hospital, Spain

${ }^{*}$ Correspondence:

Antongiulio Faggiano, Istituto di

Ricovero e Cura a Carattere

Scientifico Fondazione SDN, 80131

Napoli, Italy.

e-mail: afaggian@unina.it
Introduction: Histological distinction between typical and atypical bronchopulmonary carcinoids is based on mitotic activity and necrosis. Regardless of these two parameters, outcome after surgery is often unpredictable. In this study the prognostic value of different clinico-pathological factors was retrospectively analyzed in a large series of patients with bronchopulmonary carcinoid. Materials and Methods: The long-term post-surgical outcome of 106 radically treated patients affected by bronchopulmonary carcinoid from two Italian centers was correlated with tumor characteristics assessed by combining conventional histology with a panel of immunohistochemical markers of neuroendocrine differentiation (chromogranin-A, NSE) and proliferation activity (Ki-67 score). Results: Carcinoids were assessed as typical $(T C=75 ; 70.8 \%)$ and atypical $(A C=31 ; 29.2 \%)$. Mean follow-up was 8.3 years (range: 0-20; median: 8.0). All cases expressed neuroendocrine markers. At univariate analysis, tumor recurrence [14/75 TC (18.7\%), 15/31 AC (48.4\%)] correlated with carcinoid histotype $(P=0.003)$, tumor size $(P=0.012)$, mitotic index $(P=0.044)$, Ki-67 score $(P<0.0001)$, and synchronous node metastasis $(P=0.037)$. Of these, Cox multivariate analysis confirmed only Ki-67 score as independent predictor of disease recurrence $(P=0.009)$. The best cut-off for Ki-67 score (calculated by ROC curves) discriminating recurrent vs non-recurrent disease was $4 \%$ (sensitivity $79.3 \%$; specificity $83.8 \%$; area under the curve 0.85$)$. By stratifying patients according to this cut-off, a significantly different disease-free survival was found (log-rank test $P<0.0001$ ). Conclusion: Ki-67 score accurately separates bronchopulmonary carcinoids in two well-distinct histo-prognostic categories. Ki-67 score predicts the patients outcome better than mitotic count, histotype, and tumor stage and it is therefore helpful in establishing the appropriate follow-up.

Keywords: neuroendocrine tumors, Ki-67, tumor markers

\section{INTRODUCTION}

Bronchopulmonary carcinoids are no more than $2 \%$ of lung primary tumors (Hurt and Bates, 1984; Travis et al., 1998a; Hage et al., 2003; Modlin et al., 2003; Beasley et al., 2004; Warren and Hammar, 2006; Gustafsson et al., 2008). On the basis of their histological features (mitotic rate and presence of necrosis) they are divided in two subgroups: typical carcinoids (TC) and atypical ones (AC), these last characterized by a worse prognosis than the first subgroup (Paladugu et al., 1985; Travis et al., 1998b, 2008). In this study, histological features of the tumor have been evaluated and some immunohistochemical markers have been tested as possible prognostic factors for risk stratification of patients.
The mitotic index (number of mitosis for 10 high-power fields) is one of the criteria used for the differential diagnosis between typical and atypical forms, but given the considerable inter-operators variability, the expression of Ki-67 was tested as a potentially more accurate tool for the assessment of tumor proliferative activity. $\mathrm{Ki}-67$ is a nuclear antigen expressed by proliferating cells. In bronchopulmonary carcinoids Ki-67 score has been reported to be around $0.2-1.1 \%$ in TC and $0.3-20.3 \%$ in AC. Several studies reported an association between high Ki-67 expression and a worse prognosis (el-Naggar et al., 1991; Costes et al., 1995; Slodkowska et al., 1998; Beasley et al., 2000; Laitinen et al., 2000; Kobayashi et al., 2004; Faggiano et al., 2008; Rugge et al., 2008; Skov et al., 2008). Data available in literature show a considerable variability 
both at a clinical-therapeutic level in terms of diagnostic evaluation, treatment carried out, post-surgical evaluation and at a laboratory level in terms of sample processing and, histological evaluation. Furthermore, many studies include a small number of patients with short follow-up and do not compare Ki-67 with other prognostic factors. This retrospective analysis of a large homogeneous population of consecutive patients affected by bronchopulmonary carcinoid radically treated in two experienced centers for neuroendocrine tumors and followed for an average of 8 years aims to clarify the prognostic role of different clinico-pathological factors in the long-term post-surgical outcome.

\section{MATERIALS AND METHODS STUDY POPULATION}

One hundred thirty consecutive cases of bronchopulmonary carcinoid treated at the University Hospitals of Udine (from 1990 to 2010) and at the Cardarelli Hospital of Naples (from 2000 to 2010) were histologically reviewed and independently assessed by two expert pathologists. Tumors treated with laser therapy (13 cases, 12 typical and 1 atypical carcinoid) or surgery without an adequate sampling of regional lymph nodes (10 cases, all typical carcinoid) were excluded. A further case was excluded because at histology it was consistent with multiple lesions less than $2 \mathrm{~mm}$ in diameter, thus having to be ascribed to the category of "tumorlets." The patients finally included in the study were 106, all treated with radical surgical excision (confirmed by histological sections), and adequate sampling of the ilo-mediastinal lymph nodes (sampling lymphadenectomy). No patient underwent adjuvant therapy except in cases of disease relapse.

\section{PATHOLOGY}

Tumor stage was established on the basis of the last TNM classification of lung tumors. The gross features of the tumor were derived from the original macroscopic report. The histological features of the tumor were obtained by new sections of the paraffin-embedded samples; these sections were then stained with hematoxylin and eosin and analyzed by two expert pathologists (Carlo Alberto Beltrami, Stefano Pizzolitto; not familiar with the original diagnosis or the details of the patient) who reviewed the whole series on the basis of the recent seventh WHO classification of lung tumors (Travis et al., 2008; there was only one case of disagreement between the two pathologists and it was decided by a joint review of the sample). The histological features evaluated were: necrosis (assessed in terms of presence/absence) and mitotic index (estimated by the number of mitosis in 10 highperformance fields; $40 \times$ ). The immunohistochemical staining for the antigen Ki-67 (clone code Mib-1, Dako, M7240) was also done and its positivity was determined as a semiquantitative score (percentage of positive nuclei upon 2000 cells). Immunohistochemical staining for chromogranin-A (clone code DAK-A3, Dako, M0869, detection kit: EnvisionTM Dako K5007), NSE (clone BS/NC/VIH14, Dako, M0873, detection kit: EnvisionTM Dako K5007), and synaptophysin (polyclonal, Dako, A010, detection kit: EnvisionTM Dako K5007) was performed to ensure the neuroendocrine nature of the tumor, and resulted positive in all cases (Rusch et al., 1996).

\section{STATISTICAL ANALYSIS}

The main outcome considered in this study was disease-free survival defined as a period of time (in years) elapsed between surgery and recurrence. First of all, we performed the univariate analysis of every prognostic factor using the Mann-Whitney, Fisher exact, and Spearman's rho tests to determine statistical significance of correlation. To identify independent variables associated with outcome we used the multivariate Cox regression model. The analysis of ROC curves (Receiver Operating Characteristics) allowed us to identify the best cut-off of Ki-67 nuclear expression to stratify the patients according to the clinical outcome (relapse vs no relapse). The probability of disease-free survival was evaluated by the Kaplan-Meier curves stratifying patients according to the prognostic factors. The statistical significance of the difference between curves was evaluated by log-rank test. $P$ values lower than 0.05 were considered significant.

\section{RESULTS DEMOGRAPHIC CHARACTERISTICS OF THE POPULATION AND CLINICAL OUTCOME}

Among the 106 patients included in the study the male/female ratio was $45 / 61$ without a statistically significant difference between the group of TC and that of AC (30/45 and 15/16, respectively). The average age was $55.1 \pm 14.9$ years (range $21-82$, median 57 ) without any significant difference between TC (average 53.3 \pm 14.9 ; median 55.0) and AC (mean 59.4 \pm 14.1 ; median 63.0). The mean follow-up was $8.3 \pm 5.3$ years with a range from 0 to 20 years and a median of 8 years. Recurrence occurred in 29 patients $(27.4 \%)$, 14 of whom had a TC and 15 an AC, with a mean diseasefree survival of $7.3 \pm 5.2$ years $(8.5 \pm 5.1$ years among the patients with TC and $4.2 \pm 4.2$ years among those with AC). Twenty-two patients $(20.8 \%)$ died during the follow-up, 11 of them because of recurrence/progression of disease (Table $\mathbf{1}$ ).

\section{MACROSCOPIC FEATURES AND HISTOLOGICAL DIAGNOSIS}

The tumor size ranged from a minimum of $1 \mathrm{~cm}$ to a maximum of $10.0 \mathrm{~cm}$ with an average of $2.6 \pm 1.4 \mathrm{~cm}$ and a median of $2.2 \mathrm{~cm}$. In 55 cases $(51.9 \%)$ the tumor was centrally located (main, lobar, or segmental bronchus), while in 51 cases $(48.1 \%)$ it was peripherally located. The exact location of tumors within the lungs is shown in Figure 1. Synchronous lymph node metastases were found in $21 / 106$ cases $(19.8 \%), 14$ of these were in stage N1 and 7 in stage N2. Staging of patients according to the pTNM system showed the following results: 54 patients (50.9\%) stage IA, 29 (27.4\%) stage IB, $8(7.5 \%)$ stage IIA, $4(3.8 \%)$ stage IIB, $8(7.5 \%)$ stage IIIA, and $3(2.8 \%)$ stage IV. Of the 106 cases analyzed, $75(70.8 \%)$ cases were classified as TC and $31(29.2 \%)$ as AC. Regarding the original diagnosis, this was confirmed in 91 cases while it was changed in the remaining 15 cases. Of these, seven were originally named as "neuroendocrine tumors" (three reclassified as TC and four as $\mathrm{AC})$, two were upgraded to $\mathrm{AC}$ and six were downgraded to TC (Table 2).

Overall mitotic index was found to be an average of $1.4 \pm 1.8$ mitosis with a statistically significant difference (Mann-Whitney $P<0.0001$ ) between TC (average $0.6 \pm 0.9$ ) and AC (mean $3.4 \pm 2.0$ ). AC showed a significant association with the incidence of lymph node metastases (Fisher test $P<0.050$ ). Overall, 
Table 1 | Characteristics of the study population.

\begin{tabular}{|c|c|c|c|c|}
\hline & All 106 & TC $75(70.8 \%)$ & AC $31(29.2 \%)$ & $P$ value (TC vs $A C$ ) \\
\hline Sex (male) & $45(42.5 \%)$ & $30(40 \%)$ & $15(48.4 \%)$ & $P=0.5628$ \\
\hline Age (years) & $55.1 \pm 14.9$ & $53.3 \pm 14.9$ & $59.4 \pm 14.1$ & $P=0.0596$ \\
\hline Central Location (\%) & $55(51.9 \%)$ & $41(54.7 \%)$ & $14(45.2 \%)$ & $P=0.4982$ \\
\hline Mitosis upon $10 \mathrm{HPF}$ & $1.4 \pm 1.8$ & $0.6 \pm 0.9$ & $3.4 \pm 2.0$ & $P<0.0001$ \\
\hline $\mathrm{pN} 1(\%)$ & $14(13.2 \%)$ & $9(12.0 \%)$ & $5(16.1 \%)$ & $P=0.0490$ \\
\hline pN2 (\%) & $7(6.6 \%)$ & $3(4.0 \%)$ & $4(12.9 \%)$ & $P=0.0425$ \\
\hline Ki-67 (\%) & $5.0 \pm 10.0$ & $2.9 \pm 3.2$ & $9.5 \pm 16.6$ & $P=0.0200$ \\
\hline Recurrence (\%) & $29(27.4 \%)$ & $14(18.7 \%)$ & $15(48.4 \%)$ & $P=0.0039$ \\
\hline DFS (years) & $7.3 \pm 5.2$ & $8.5 \pm 5.1$ & $4.2 \pm 4.2$ & $P<0.0001$ \\
\hline
\end{tabular}
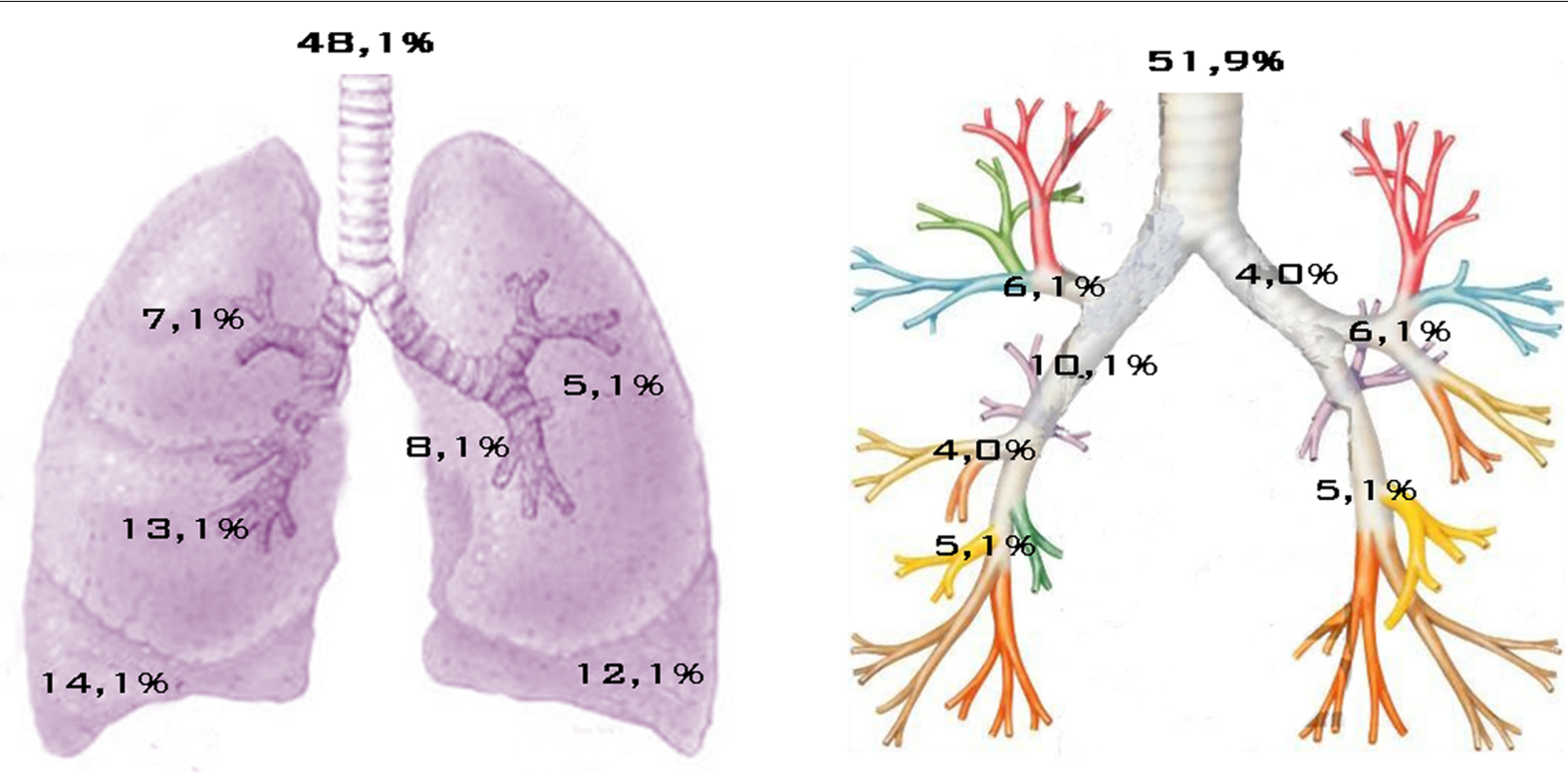

FIGURE 1 | Location of carcinoids within the lungs.

the nuclear expression of Ki-67 was found to be $5.0 \pm 10.0 \%$ (median $2.1 \%$ ) with a statistically significant correlation with the mitotic index (Spearman's rho $P=0.039$ ). Ki-67 score was also significantly higher in AC than TC (Mann-Whitney $P=0.020)$.

\section{PROGNOSTIC FACTORS}

At univariate analysis, the recurrence of disease was significantly correlated to histotype (Fisher test $P=0.003$ ), tumor size (Mann-Whitney $P=0.012$ ), pTNM stage (Fisher $P=0.032$ ), presence of lymph node metastases (Fisher $P=0.028$ ), $\mathrm{N}$ stage (Fisher $P=0.014$ ), mitotic index (Mann-Whitney $P=0.044$ ), and Ki-67 score (Mann-Whitney $P=0.0002$ ). However, there was no significant correlation between the risk of relapse and age (Mann-Whitney $P=0.944$ ) or gender (Fisher $P=0.272$; Table 3).

All possible prognostic factors identified by univariate analysis were tested at the multivariate analysis according to Cox regression model which showed that the best independent variable being able to predict recurrence of disease was Ki-67 score (risk ratio 20.5; 95\% CI: 2.1-201.3\%; $P=0.009)$. The best cut-off for Ki67 score to identify those patients at high risk of recurrence was determined by ROC curves analysis: the cut-off obtained was $4 \%$ with a sensitivity of $79.3 \%$ (95\% CI: $60.3-92.0 \%)$, a specificity 
Table 2 | Characteristics of the 15 reclassified cases.

\begin{tabular}{lll}
\hline \multicolumn{1}{c}{ Original diagnosis } & \multicolumn{2}{c}{ Revision } \\
\cline { 2 - 3 } & TC & AC \\
\hline "Neuroendocrine tumor" & 3 & 4 \\
TC & $/$ & 2 \\
AC & 6 & $/$ \\
Total & $9(60.0 \%)$ & $6(40.0 \%)$ \\
Nodal metastasis (\%) & 0 & $1(6.7 \%)$ \\
Relapse (\%) & 0 & $4(26.7 \%)$
\end{tabular}

Table 3 | Clinico-pathological prognostic factors evaluated at the univariate and multivariate analysis.

\begin{tabular}{lllll}
\hline $\begin{array}{l}\text { Univariate analysis. Correlation } \\
\text { between recurrence and: }\end{array}$ & \multicolumn{3}{c}{$\begin{array}{c}\text { Multivariate analysis } \\
\text { (Cox regression model) }\end{array}$} \\
\cline { 3 - 5 } & & Risk ratio & $\mathbf{9 5 \%} \mathbf{~ C l}$ & $\boldsymbol{P}$ \\
\hline Age & $P=0.944$ (NS) & $/$ & $/$ & $/$ \\
Sex & $P=0.272$ (NS) & $/$ & $/$ & $/$ \\
Size & $P=0.012$ & 1.0 & $0.9-1.1$ & 0.502 \\
Histotype & $P=0.003$ & 3.4 & $1.1-10.7$ & 0.061 \\
(TC vs AC) & & & & \\
Mitotic index & $P=0.044$ & 0.9 & $0.7-1.3$ & 0.585 \\
Nodal metastasis & $P=0.028$ & 2.3 & $0.8-6.6$ & 0.110 \\
N stage & $P=0.014$ & 0.9 & $0.2-3.1$ & 0.822 \\
pTNM stage & $P=0.032$ & $/$ & $/$ & $/$ \\
Ki-67 & $P=0.0002$ & 20.5 & $2.1-201.3$ & 0.009 \\
Ki-67 $>0.04$ & $P=0.000000006$ & $/$ & $/$ & $/$ \\
& & & & \\
\hline
\end{tabular}

of $83.8 \%$ (95\% CI: $72.9-91.6 \%$ ), and an area under the curve of 0.85 (95\% CI: 0.76-0.91\%; Figure 2). Stratifying patients according to the Ki-67 cut-off of $4 \%$ results in a statistically significant difference in disease-free survival (log-rank test $P<0.0001$; Figure 3).

\section{DISCUSSION}

According to the recent classification of lung tumors developed by the World Health Organization (Travis et al., 2008) it is possible to divide bronchopulmonary carcinoids in two subgroups (TC and AC) on the basis of two main histologic features: the presence or absence of necrosis and the number of mitosis per 10 high-performance fields. The TC has a 5-year survival between 87 and $100 \%$ and a 10-years survival between 82 and $92 \%$ in different studies. AC has a 5-year survival between 56 and 78\% and a 10-years survival between 35 and 67\% (Travis et al., 1998a; Beasley et al., 2000; Cooper et al., 2001; Hage et al., 2003; GarcíaYuste et al., 2007). Previous studies are often based on small or non-homogeneous series with high variability in terms of presurgical evaluation, surgical management, histological processing of samples, and adjuvant therapy when given. Moreover many studies have a small number of cases and a short follow-up (Costes et al., 1995; Soga and Yakuwa, 1999; Ferguson et al., 2000; Laitinen et al., 2000; Cooper et al., 2001; Fink et al., 2001; García-Yuste et al., 2007). In this study, the inclusion of patients from two institutions,

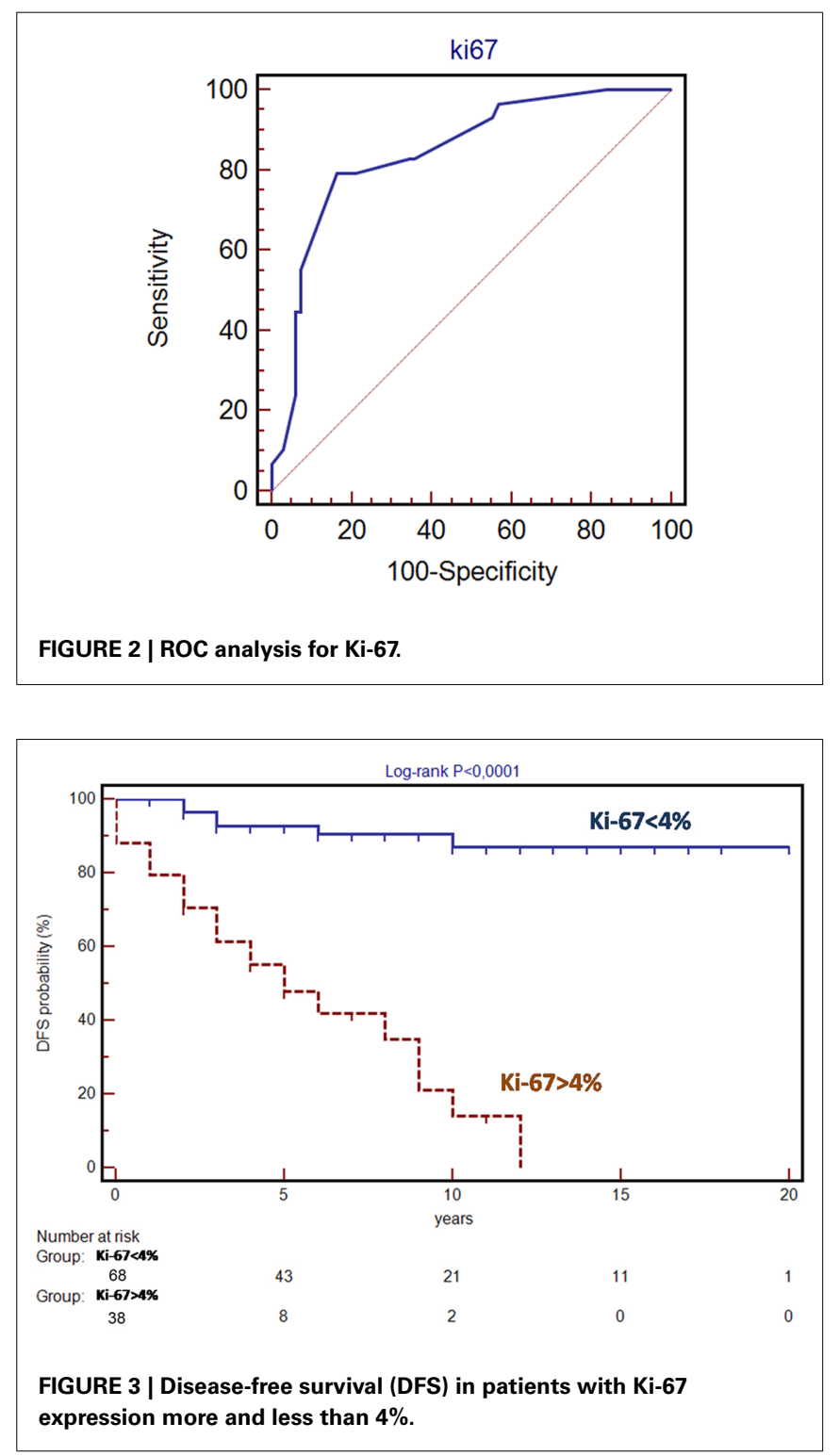

all undergone the same surgical approach and whose histologic samples were analyzed with the same procedures and reviewed by two pathologists, helped to reduce inter-center variability. Moreover, the exclusion of patients who had adjuvant post-surgical therapy allowed us to eliminate a confounding factor influencing the patients outcome. The ratio between TC and AC (75:31) found in our series is slightly higher than that found in other series. This may be partly explained by the fact that many of the excluded cases were TC. It should also be noted that the percentage of atypical carcinoid in published series shows a considerable variability (1019\%; Soga and Yakuwa, 1999; Ferguson et al., 2000; Fink et al., 2001).

The ratio between central and peripheral forms (55:51) is instead consistent with data reported in the literature (Soga and Yakuwa, 1999; Ferguson et al., 2000; Fink et al., 2001). Moreover the evidence of a higher mitotic index, higher incidence of synchronous node metastases and higher risk of relapse found in 


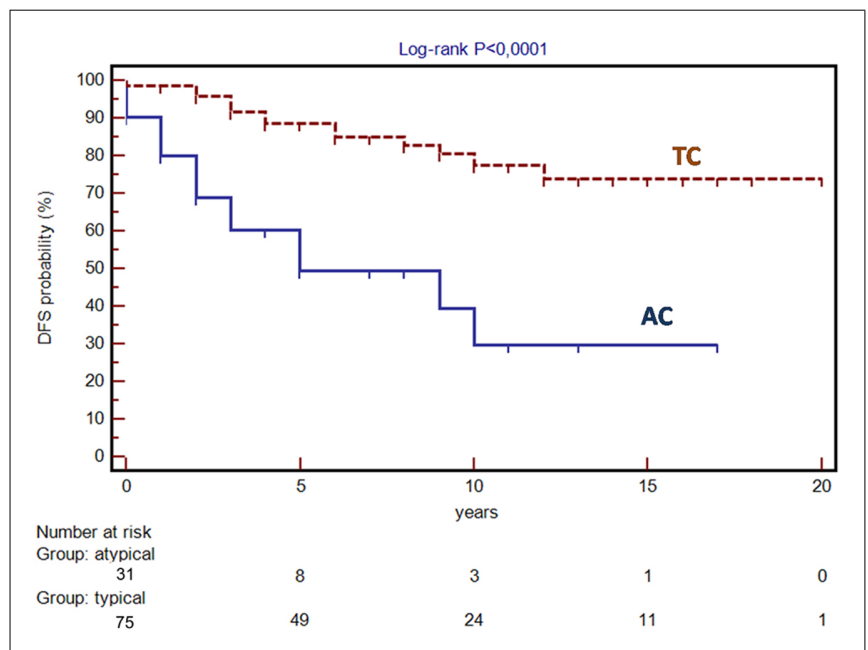

FIGURE 4 | Disease-free survival (DFS) in patients with typical (TC) and atypical carcinoid (AC).

AC than TC is consistent with previous observations (Soga and Yakuwa, 1999; Ferguson et al., 2000; Fink et al., 2001), as highlighted by the difference between the disease-free survival curves (log-rank $P<0.0001$; Figure 4). With regard to long-term survival, it appears to be $100 \%$ at 5,10 , and 15 years for TC and 65,59 , and $44 \%$ at 5,10 , and 15 years for AC. As shown by the fact that Kaplan-Meier curves tend to reach a plateau only after 15-20 years follow-up, carcinoids are tumors that may recur even after many years after surgery and therefore require a very long follow-up.

The univariate analysis identified factors that are related with the risk of relapse (histological diagnosis, mitotic index, lymph node metastases, size of primary tumor, TNM stage, and expression of Ki-67), but their prognostic potential disappeared when the expression of Ki-67 was added in Cox's multivariate regression model. The relationship between tumor proliferative activity and outcome is a given, but there are still doubts as to what is the best method to determine the proliferative activity of tumor, what is the most appropriate cut-off in predicting the outcome and whether the proliferative rate constitutes an independent variable in predicting the outcome.

\section{REFERENCES}

Arbiser, Z. K., Arbiser, J. L., Cohen, C., and Gal, A. A. (2001). Neuroendocrine lung tumors: grade correlates with proliferation but not angiogenesis. Mod. Pathol. 14, 1195-1199.

Beasley, M. B., Thunnissen, F. B., Brambilla, E., Hasleton, P., Steele, R., Hammar, S. P., Colby, T. V., Sheppard, M., Shimosato, Y., Koss, M. N., Falk, R., and Travis, W. D. (2000). Pulmonary atypical carcinoid: predictors of survival in 106 cases. Hum. Pathol. 31, 1255-1265.

Travis et al. (1998b) set the cut-off for the distinction between $\mathrm{TC}$ and $\mathrm{AC}$ in a mitotic rate of 2 mitoses per 10 high-performance fields; this value was then adopted in all subsequent publications as well as in the recent WHO classification of lung tumors (Travis et al., 1998a,b, 2008; Beasley et al., 2004). When proliferative activity is assessed by the expression of $\mathrm{Ki}-67$, there are some important differences in the distinction between TC and AC. In the study by Costes et al. (1995) including 47 cases of carcinoid (31 TC and $16 \mathrm{AC}$ ) it was found that in TC expression of Ki-67 covered less than $1 \%$ of the nuclei, while in $\mathrm{AC}$ the positivity rate was significantly higher (average 2.4\%). Arbiser et al. (2001) evaluated the proliferative activity of carcinoid counting the number of Ki-67 positive nuclei in 1000 cells showing fewer positive nuclei in TC than AC carcinoids, but this difference was not found to be statistically significant. In 31 other cases of carcinoid (21 TC and $10 \mathrm{AC}$ ), Laitinen et al. (2000) found less than 1\% of Ki-67 positive nuclei in 28 of 31 cases and showed a significant association between a high percentage of positive nuclei (10-20\%) and diagnosis of AC. In our population the expression of $\mathrm{Ki}-67$ is an average of $2.9 \%$ for TC and $9.5 \%$ in AC. Moreover, multivariate analysis showed that there was a statistically significant correlation between the expression of Ki-67 and the long-term outcome. The cut-off of $4 \%$ (Receiver Operating Characteristic analysis) was able to distinguish between patients at high and at low risk of recurrence with high sensitivity and specificity (respectively 79.3 and $83.8 \%$ ), providing an essential element in prognostic stratification of patients.

In the present study the evaluation of Ki-67 was made by counting the percentage of positive nuclei upon 2000 cells. At the moment the operator counting is the gold standard, however there are increasing evidences about the effectiveness of computerized counting systems that in the future can be used in order to decrease inter-operator variability and analysis time. (Fasanella et al., 2011; Slodkowska et al., 2011).

In conclusion this study has shown that the expression of Ki67 could be an optimal prognostic factor for bronchopulmonary carcinoids, and therefore, Ki-67 score may be a crucial factor to decide the postoperative follow-up strategies.

\section{ACKNOWLEDGMENTS}

This study was partially supported by a grant from the Italian Minister of Research and University in Rome (no. 2008LFK7J5).

Costes, V., Marty-Ané, C., Picot, M. C. Serre, I., Pujol, J. L., Mary, H., and Baldet, P. (1995). Typical and atypical bronchopulmonary carcinoid tumors: a clinicopathologic and KI67-labeling study. Hum. Pathol. 26, 740-745.

el-Naggar, A. K., Ballance, W., Karim, F. W., Ordóñez, N. G., McLemore, D., Giacco, G. G., and Batsakis, J. G. (1991). Typical and atypical bronchopulmonary carcinoids. A clinicopathologic and flow cytometric study. Am. J. Clin. Pathol. 95, 828-834.
Faggiano, A., Mansueto, G., Ferolla, P., Milone, F., del Basso de Caro, M. L., Lombardi, G., Colao, A., and De Rosa, G. (2008). Diagnostic and prognostic implications of the World Health Organization classification of neuroendocrine tumors. J. Endocrinol. Invest. 31, 216-223.

Fasanella, S., Leonardi, E., Cantaloni, C., and Eccher, C. (2011). Proliferative activity in human breast cancer: Ki-67 automated evaluation and the influence of different Ki-67 equivalent antibodies. Diagn. Pathol. 6(Suppl. 1), S7. 
Ferguson, M. K., Landreneau, R. J., Hazelrigg, S. R., Altorki, N. K., Naunheim, K. S., Zwischenberger, J. B., Kent, M., and Yim, A. P. (2000). Long-term outcome after resection for bronchial carcinoid tumors. Eur. J. Cardiothorac. Surg. 18, 156-161.

Fink, G., Krelbaum, T., Yellin, A., Bendayan, D., Saute, M., Glazer, M., and Kramer, M. R. (2001). Pulmonary carcinoid: presentation, diagnosis, and outcome in 142 cases in Israel and review of 640 cases from the literature. Chest 119, 1647-1651.

García-Yuste, M., Matilla, J. M., Cueto, A., Paniagua, J. M., Ramos, G., Cañizares, M. A., Muguruza, I., and Spanish Multi-centric Study of Neuroendocrine Tumours of the Lung for the Spanish Society of Pneumonology and Thoracic Surgery (EMETNE-SEPAR). (2007). Typical and atypical carcinoid tumours: analysis of the experience of the Spanish Multi-centric Study of Neuroendocrine Tumours of the Lung. Eur. J. Cardiothorac. Surg. 31, 192-197.

Gustafsson, B. I., Kidd, M., Chan, A., Malfertheiner, M. V., and Modlin, I. M. (2008). Bronchopulmonary neuroendocrine tumors. Cancer 113, 5-21.

Hage, R., de la Rivière, A. B., Seldenrijk, C. A., and van den Bosch, J. M. (2003). Update in pulmonary carcinoid tumors: a review article. Ann. Surg. Oncol. 10, 697-704.

Hurt, R., and Bates, M. (1984). Carcinoid tumours of the bronchus: a 33 year experience. Thorax 39, 617-623.

Kobayashi, Y., Tokuchi, Y., Hashimoto, T., Hayashi, M., Nishimura, H.,
Ishikawa, Y., Nakagawa, K., Sato, Y., Takahashi, A., and Tsuchiya, E. (2004). Molecular markers for reinforcement of histological subclassification of neuroendocrine lung tumors. Cancer Sci. 95, 334-341.

Laitinen, K. L., Soini, Y., Mattila, J., and Pääkkö, P. (2000). Atypical bronchopulmonary carcinoids show a tendency toward increased apoptotic and proliferative activity. Cancer 88, 1590-1598.

Modlin, I. M., Lye, K. D., and Kidd, M. (2003). A 5-decade analysis of 13,715 carcinoid tumors. Cancer 97, 934-959.

Paladugu, R. R., Benfield, J. R., Pak, H. Y., Ross, R. K., and Teplitz, R. L. (1985). Bronchopulmonary Kulchitsky cell carcinomas. A new classification scheme for typical and atypical carcinoids. Cancer 55, 1303-1311.

Rugge, M., Fassan, M., Clemente, R., Rizzardi, G., Giacomelli, L., Pennelli, G., Mescoli, C., Segat, D., and Rea, F. (2008). Bronchopulmonary carcinoid: phenotype and long-term outcome in a single-institution series of Italian patients. Clin. Cancer Res. 14, 149-154.

Rusch, V. W., Klimstra, D. S., and Venkatraman, E. S. (1996). Molecular markers help characterize neuroendocrine lung tumors. Ann. Thorac. Surg. 62, 798-809; discussion 809-810.

Skov, B. G., Krasnik, M., Lantuejoul, S., Skov, T., and Brambilla, E. (2008). Reclassification of neuroendocrine tumors improves the separation of carcinoids and the prediction of survival. J. Thorac. Oncol. 3, 1410-1415.
Soga, J., and Yakuwa, Y. (1999). Bronchopulmonary carcinoids: an analysis of1, 875 reported cases with special reference to a comparison between typical carcinoids and atypical varieties. Ann. Thorac. Cardiovasc. Surg. 5, 211-219.

Slodkowska, J., Langfort, R., Rudzinski, P., and Kupis, W. (1998). Typical and atypical pulmonary carcinoids - pathologic and clinical analysis of 77 cases. Pneumonol. Alergol. Pol. 66, 297-303.

Slodkowska, J., Markiewicz, T., and Grala, B. (2011). Accuracy of a remote quantitative image analysis in the whole slide images. Diagn. Pathol. 6(Suppl. 1), S20.

Travis, W. D., Giroux, D. J., Chansky, K., Crowley, J., Asamura, H., Brambilla, E., Jett, J., Kennedy, C., Rami-Porta, R., Rusch, V. W., Goldstraw, P., and International Staging Committee and Participating Institutions. (2008). The IASLC Lung Cancer Staging Project: proposals for the inclusion of bronchopulmonary carcinoid tumors in the forthcoming (seventh) edition of the TNM Classification for Lung Cancer. J. Thorac. Oncol. 3, 1213-1223.

Travis, W. D., Rush, W., Flieder, D. B., Falk, R., Fleming, M. V., Gal, A. A., and Koss, M. N. (1998a). Survival analysis of 200 pulmonary neuroendocrine tumors with clarification of criteria for atypical carcinoid and its separation from typical carcinoid. Am. J. Surg. Pathol. 22, 934-944.

Travis, W. D., Gal, A. A., Colby, T. V., Klimstra, D. S., Falk, R., and Koss, M. N. (1998b). Reproducibility of neuroendocrine lung tumor classification. Hum. Pathol. 29, 272-279.

Warren, W. H., and Hammar, S. P. (2006). The dispersed neuroendocrine system, its bronchopulmonary elements, and neuroendocrine tumors presumed to be derived from them: myths, mistaken notions, and misunderstandings. Semin. Thorac. Cardiovasc. Surg. 18, 178-182.

Conflict of Interest Statement: The authors declare that the research was conducted in the absence of any commercial or financial relationships that could be construed as a potential conflict of interest.

Received: 23 June 2011; accepted: 30 July 2011; published online: 19 August 2011.

Citation: Grimaldi F, Muser D, Beltrami CA, Machin P, Morelli A, Pizzolitto S, Talmassons G, Marciello $F$ Colao AAL, Monaco $R$, Monaco $G$ and Faggiano $A$ (2011) Partitioning of bronchopulmonary carcinoids in two different prognostic categories by $\mathrm{Ki}$ 67 score. Front. Endocrin. 2:20. doi: 10.3389/fendo.2011.00020

This article was submitted to Frontiers in Cancer Endocrinology, a specialty of Frontiers in Endocrinology.

Copyright (c) 2011 Grimaldi, Muser, Beltrami, Machin, Morelli, Pizzolitto, Talmassons, Marciello, Colao, Monaco, Monaco and Faggiano. This is an openaccess article subject to a non-exclusive license between the authors and Frontiers Media SA, which permits use, distribution and reproduction in other forums, provided the original authors and source are credited and other Frontiers conditions are complied with. 